\title{
Electronic Structure Effects in Liquid Water studied by Photoelectron Spectroscopy and Density Functional Theory
}

\author{
D. Nordlund ${ }^{1,2}$, M. Odelius ${ }^{2}$, H. Bluhm ${ }^{3}$, H. Ogasawara ${ }^{1}$, L.G.M. Pettersson ${ }^{2}$, A. Nilsson ${ }^{1,2^{*}}$
}

1 Stanford Synchrotron Radiation Laboratory, 2575 Sand Hill Road, Menlo Park, CA 94025, US

2 FYSIKUM, AlbaNova University Center, Stockholm University, S-106 91 Stockholm, Sweden

3 Lawrence Berkeley National Laboratory, Chemical Sciences Division, Berkeley, California 94720

* Corresponding author: nilsson@ slac.stanford.edu

We present valence photoelectron emission spectra of liquid water in comparison with gas-phase water, ice close to the melting point, low temperature amorphous and crystalline ice. All aggregation states have major electronic structure changes relative to the free molecule, with rehybridization and development of bonding and anti-bonding states accompanying the hydrogen bond formation. Sensitivity to the local structural order, most prominent in the shape and splitting of the occupied $3 a_{1}$ orbital, is understood from the electronic structure averaging over various geometrical structures, and reflects the local nature of the orbital interaction. 


\section{Introduction}

The unique properties of liquid water and ice are connected to the hydrogen bond $(\mathrm{H}-$ bond) which ultimately defines the physical properties of the condensed phases of water. In order to understand the nature of the hydrogen bond, it is important to derive a microscopical picture that effectively can describe the molecular origin of the chemical interaction between the water molecules in the liquid. Of particular importance is whether the electrostatic picture of hydrogen bonding (2) including charge redistribution within the molecule induced by the field of the neighboring molecules (3-6) gives an accurate representation of the chemical interaction, as this is the basis for many classical molecular dynamics force field descriptions of liquid water (6-9). The contrary view holds that the H-bond involves significant charge redistribution in the valence electronic structure that differs from that induced by the electrostatic field of the surrounding molecules (10-14). To this end, quantum mechanical ab initio methods have been used, in particular to address the cooperativity in hydrogen-bonded aggregates (10), showing nonpairwise additive effects in disagreement with the simple electrostatic picture (14-17). To better understand the fundamental nature of the hydrogen bond in water it is essential to investigate the liquid experimentally, using techniques such as photoelectron emission (PE) (18-20) and X-ray emission (XE) (21) that are sensitive to the electronic structure and allow an orbital picture interpretation. Besides studies of gas-phase water (22) and water clusters (23), the comparison of the electronic structure of liquid water to H-bonded model systems such as ice $(13,24,25)$, for which the H-bonding network is relatively well known, is of particular importance.

The valence PE spectra from water vapor and ice were shown already in the 1960s in the work by Kai Siegbahn and co-workers (22). Early PE studies on ice $(24,25)$ showed major electronic structure changes relative to the gas-phase, including binding energy shifts and band broadening. Mårtensson et al. (24) reported a rather unperturbed lone pair orbital in ice and a delocalized $3 \mathrm{a}_{1}$, associated with strong orbital overlap between neighboring $3 \mathrm{a}_{1}$ orbitals resulting in split bands, corroborating early electronic structure calculations (26). The splitting of the $3 \mathrm{a}_{1}$ state in ice $(24,25)$ and other associated electronic structure effects $(26,27)$, were further investigated in a recent study, which also discussed the general electronic and energetic contributions to the hydrogen bond formation (13). Using PE and $\mathrm{X}$-ray absorption (XA) spectroscopy in combination with density functional theory (DFT) (13), it was shown that the hydrogen bond involves charge donation from the lone-pair to the $\mathrm{OH}$ antibonding orbitals on neighboring molecules, which results in a lowering of the repulsion, in line with the charge transfer picture of hydrogen bonding that is derived from natural bond orbital analysis $(11,12)$.

The application of PE to aqueous solutions was pioneered by H. Siegbahn and coworkers who overcame experimental difficulties with electron detection to measure liquids with modest vapor pressures (18). As for other early approaches the application to neat water was hindered by the exceptionally high vapor pressure ( 4.6 torr near the melting point). To that end, a very thin liquid jet (microjet) was developed by Faubel and coworkers for measuring liquid water in a vacuum environment (28), used initially with HeI excitation (28) and later with synchrotron radiation (19). Valence band PES from liquid water microjets $(19,20)$ have reported accurate binding energies and cross-sections, full 
widths and experimental gas-to-liquid energy shifts. An alternative to the microjet technique is to acquire PE spectra in gas environments at pressures up to several torr using a differentially pumped electrostatic lens (29), allowing studies of thin films of liquid water grown and supported on a solid in equilibrium with the vapor pressure (30).

The electronic structure of liquid water and ice has also been studied at many different levels of theory, in both periodic and cluster calculations (26,27,31-34). In early tight-binding calculations of ice (26), the energy dispersion in the Bloch orbitals and density of states were calculated and splittings in the valence states arising from molecular states of $a_{1}$ symmetry were observed. In particular, a $1 \mathrm{eV}$ splitting in the $3 \mathrm{a}_{1}$ state was predicted, in contrast to the small dispersion of the $b_{1}$ and $b_{2}$ states. More recently, density functional theory in combination with molecular dynamics simulations, which enable studies at finite temperature, has been employed to study also liquid water $(33,34)$. The width of the bands in the liquid is reported to have significant contributions $(\sim 0.5 \mathrm{eV})$ from thermal broadening, although, in particular for the broad $3 \mathrm{a}_{1}$ state, the electronic interaction is dominating (33). Moreover, the electronic structure of liquid water is relatively insensitive to the long-range structural order/disorder (34).

Here we present valence PE spectra of liquid water, which we compare to those of amorphous ice, crystalline ice at two different temperatures, and gas-phase water. By using different excitation energies $(100 \mathrm{eV}$ and $530 \mathrm{eV})$, the relative cross-section between oxygen $2 \mathrm{~s}$ - and 2p-like orbitals is varied experimentally, in analogy to Ref (13). The high $3 \mathrm{a}_{1}$ cross-section at $530 \mathrm{eV}$ allows a direct comparison of the $3 \mathrm{a}_{1}$ spectral shape between the aggregation states, unattainable in previous studies of liquid water $(19,21)$. The major electronic structure changes relative to the free molecule are similar in liquid water and all ice samples, showing that rehybridization and formation of bonding and anti-bonding states are integral parts of the hydrogen bond. The experimental spectra display non-specific sensitivity to the local structural order, most prominently in the shape and splitting of the occupied $3 a_{1}$ orbital between the different aggregation states. This finding is supported by theoretical calculations of various structural motifs, using DFT to represent the valence photoelectron cross-section at high photon energy in an atomistic way by projecting the valence states onto the oxygen core orbital.

\section{Methods}

The experiments on liquid water and ice close to the melting point were performed using the ambient pressure photoemission endstation at the undulator beamline 11.0.2 at the Advanced Light Source in Berkeley, CA (35). A liquid water droplet (volume $\sim 5 \mathrm{~mm}^{3}$ ) is condensed onto an Au substrate which is held at a temperature of $\sim 1^{\circ} \mathrm{C}$ (controlled by a Peltier element). The water drop is in thermodynamical equilibrium with its vapor, i.e. the background vapor pressure in the analysis chamber is $\sim 5$ Torr. Ice samples are prepared by condensing water onto the Au substrate at temperatures below the freezing point. The ice temperature in our measurements was kept at about $-23{ }^{\circ} \mathrm{C}$ (with $\mathrm{p}_{\text {vapor }}$ $\sim 0.58$ Torr), in order to avoid the presence of a liquid-like layer at the ice surface (36). The endstation is equipped with a differentially pumped electrostatic lens system that transfers the electrons from the sample surface (at $\sim$ Torr pressure) to the entrance slit of a Specs Phoibos 150 hemispherical electron spectrometer that is kept in high vacuum (29). Both 
the photon resolution and the analyzer resolution were kept below $150 \mathrm{meV}$, resulting in a total resolution of about $200 \mathrm{meV}$. By using a positive bias $(150 \mathrm{~V})$ on the sample, the gas-phase signal was experimentally shifted to lower kinetic energies and smeared out (37). This low kinetic energy background, along with the inelastically scattered liquid water signal due to the elevated gas-phase pressure, was removed by subtracting a Shirley-type background prior to the area normalization. Using a Gaussian function, the $1 \mathrm{~s}$ direct photoemission channel resulting from $2^{\text {nd }}$ order radiation from the grating in the $530 \mathrm{eV}$ measurements was subtracted from all spectra. For the gas-phase water experiments the pressure was kept at 0.7 Torr.

The experiments on amorphous and crystalline ice were performed at the elliptically polarized undulator beamline 5-1 at the Stanford Synchrotron Radiation Laboratory (SSRL). The UHV surface science endstation (base pressure $<1 \times 10^{-10}$ Torr) is equipped with a Scienta SES-100 hemispherical electron analyzer, a Hiden HAL-201-RC quadrupole mass spectrometer and a Bruker IR-Cube FT-IR system. The ice films were grown on a $\operatorname{Pt}(111)$ single crystal, mounted on a liquid nitrogen cooled rod with $6^{\circ}$ grazing incidence relative to the incoming photons. Cycles of $\mathrm{Ne}$ ion sputtering, electron bombardment heating $(900 \mathrm{~K})$ and $\mathrm{O}_{2}$ treatment were repeated until a clean $\mathrm{Pt}(111)$ surface was obtained (checked by PES and LEED). An 80 layers (bilayers) thick ( 225 $\AA$ ) amorphous ice film was grown at $\sim 100 \mathrm{~K}$ on the clean $\mathrm{Pt}(111)$ surface at a deposition rate of $\sim 0.08$ layers/s. Crystalline ice was obtained by isothermal heating of the amorphous ice at $150 \mathrm{~K}$, monitored by temperature programmed desorption and confirmed by IR absorption spectroscopy in the O-H stretch region (for more details on the crystalline ice preparation, see Ref (38)). In all experiments, impurities in the distilled water were removed by repeated cycles of freezing, pumping and melting prior to leaking into the experimental chamber.

Spectrum calculations were performed for different liquid water structural motifs sampled over ground state Car-Parrinello MD (CPMD) simulations (39) using the CPMD3.3 program (40). The simulations were performed in periodic boundary conditions using norm-conserving pseudopotentials in the Kleinman-Bylander form (41) with cutoff radii set to 1.05 a.u. for both the $\mathrm{s}$ and $\mathrm{p}$ channels as in our previous work (see e.g. Ref $(42,43)$ and references therein). The pseudopotential for oxygen was of Troullier-Martin type (44), nonlocal in the $l=0$ channel. For hydrogen, a local pseudopotential was parameterized with one Gaussian function (P. Giannozzi, unpublished)). The gradientcorrected B-LYP functional $(45,46)$ was used together with a plane-wave expansion of the Kohn-Sham orbitals with an energy cutoff of $70 \mathrm{Ry}$; only the Gamma-point was considered (34).

The ice and liquid water models were from our previous work $(42,43,47)$, with exception of the configuration from the 128 molecule CPMD simulation of liquid water, which was kindly supplied by the authors of Ref. (48). In analogy with Ref (42), MD simulations were not only used for spectral averaging, but also to obtain ensembles of representative structures within a local structure motif. Using these structural ensembles, single PE spectra and averages representative of the different motifs were calculated using the technique outlined in Ref (42). 
The PE spectra were decomposed into individual atomic contributions by projecting the local valence $2 p$ character (in a transition similar to $x$-ray emission) and the local valence 2 s character (via a simple $r^{2}$ operator calibrated to the 2 s content in free oxygen, 2e-) onto the spatially well-localized core orbital. This technique has the advantage of a direct measure of atomic populations, and is justified by the fact that for valence band PES at high photon energies, the ionization event occurs deep inside the atom and thereby orbitals from which the excitation occurs become atomic-like (13).

\section{Results and Discussion}

Figure 1 shows valence PE spectra of gas-phase water, liquid water and crystalline ice using $100 \mathrm{eV}$ and $530 \mathrm{eV}$ excitation energy, where the peaks are denoted according to the molecular orbitals (MO) of gas-phase water; the $1 b_{1}$ peak corresponds to the non-bonding out-of-plane lone pair orbital, the $3 \mathrm{a}_{1}$ peak corresponds to the slightly bonding mixed $\mathrm{O} 2 \mathrm{p} / \mathrm{O} 2 \mathrm{~s} / \mathrm{H} 1 \mathrm{~s}$ orbital, while the $1 \mathrm{~b}_{2}$ peak is due to an $\mathrm{O}-\mathrm{H}$ bonding $2 \mathrm{p}$-like orbital. All spectra are energy normalized to the lone pair of the crystalline ice spectrum and plotted on a binding energy scale relative to the $\mathrm{Pt}(111)$ Fermi level. The insert shows the atomic oxygen $2 \mathrm{~s}$ and $2 \mathrm{p}$ photoionization cross-section as a function of incident photon energy (reproduced from Ref (49)), showing a strong relative variation that is exploited in this study; a high $\mathrm{O} 2 \mathrm{p}$ cross section is obtained at $100 \mathrm{eV}$, whereas $530 \mathrm{eV}$ excitation energy gives a high $\mathrm{O} 2 \mathrm{~s}$ cross-section. As the mean free path of the photoelectron varies from $13 \AA$ to $\sim 20 \AA$ at the higher excitation energy (1), the fraction from the topmost surface layer (taken to be $3.5 \AA$ ) varies from about $24 \%$ to $16 \%$. In light of the relatively small spectral contrast between different local H-bonding structures in the PE spectra (i.e. between ice and water in Fig. 1 and for different H-bond configurations in Fig. 4), the effect of the surface contribution in terms of peak position and peak broadening in the analysis is not so significant (e.g. imagine the extreme case, mixing in $16 \%$ of the ice spectrum in liquid water or vice versa). Also, along the same lines, the significance of the additional surface contribution (about 8\%) in the $100 \mathrm{eV}$ excitation spectra when comparing to the $530 \mathrm{eV}$ excitation spectra is only minor if not negligible. If anything, the $530 \mathrm{eV}$ spectra are slightly more bulk sensitive than the $100 \mathrm{eV}$ spectra.

The low energy excitation spectra $(100 \mathrm{eV})$ are already reported in the literature $(13,19)$ and are shown here only for comparison since it is measured under similar conditions as the $530 \mathrm{eV}$ spectra. Since all relative peak positions (e.g. $1 \mathrm{~b}_{1}$ relative to $\left.1 \mathrm{~b}_{2}\right)$ and peak broadening (fwhm) are comparable within $0.1 \mathrm{eV}$ of previously published data (see e.g. table 2 in Ref (19)), for a thorough discussion of absolute binding energies, relative peak intensities as well as peak broadening and energy shifts between gas-phase and liquid water, we refer to the state-of-the-art PES study of liquid water reported by B. Winter et al. (19), which also discusses earlier ice and liquid water results. As reported in Ref (19) and observed in earlier studies of both ice and liquid water $(13,19,20,24,25,28)$, hydrogen bonding leads to a broadening of all spectral features resulting from final state effects and orbital overlap and $e . g$. the overall $3 \mathrm{a}_{1}$ intensity is shifted to higher binding energy.

We shall in the following focus on the novel aspects in the experiments, namely the $530 \mathrm{eV}$ excitation energy spectra of liquid water and various ice preparations with 
enhanced $3 a_{1}$ cross-section. In crystalline ice, the $3 a_{1}-3 a_{1}$ spatial and energetic overlap between neighboring molecules leads to a major energy splitting that is clearly resolved in the $530 \mathrm{eV}$ spectrum (13). Unconstrained fitting of the $3 \mathrm{a}_{1}$ intensity by two Gaussian peaks yields a split of about $1.55 \mathrm{eV}$. A similar splitting of the $3 \mathrm{a}_{1}$ state of liquid water has previously been suggested based on peak fitting of PES (19) and XES (21) data. Following B. Winter et al. (see Fig 4 in Ref (19)), we can also fit the $3 \mathrm{a}_{1}$ state of the 100 $\mathrm{eV}$ liquid water spectrum using 2 peaks with reasonably low chi square, but the confidence interval of these fitted peaks is very large and the extracted information is only meaningful with strong constraints. The real information content in the experimental low energy excitation spectra regarding $3 \mathrm{a}_{1}$ is thus very limited (we also note that the gas-phase subtraction in Ref (19) might impose further limitations on the accuracy of the curve fitting). The information content regarding the $3 \mathrm{a}_{1}$ state in the XES data (21) suffers from spectral changes associated with core-hole dynamics discussed elsewhere (42). In the high O2s cross-section data presented here, the enhanced $3 \mathrm{a}_{1}$ state (and the suppression of the gas-phase signal, see methods) allows for a direct comparison of the $3 a_{1}$ spectral shape, unattainable in previous PES (19) and XES (21) data on liquid water. In contrast to previously assumed, we observe no $3 \mathrm{a}_{1}$ splitting in the $530 \mathrm{eV}$ spectra of liquid water, but the $3 \mathrm{a}_{1}$ peak is exceptionally broad, indicating a similarly strong electronic structure change as in ice. Since the $1 b_{1}$ and $1 b_{2}$ retain the same width as for the $100 \mathrm{eV}$ excitation (fitted values within $0.1 \mathrm{eV}$ of table 2, Ref (19)), we can attribute the novel information to the enhancement of the $3 \mathrm{a}_{1}$ state through the strong $\mathrm{O} 2 \mathrm{~s}$ cross section. For example, the slightly narrower overall $3 a_{1}$ width in Ref (19) (table 2 ) is based on 2 fitted Gaussians and is not experimentally well defined. Is the splitting of the $3 a_{1}$ orbital specific to the tetrahedral structure in crystalline ice, or is it an integral part of the hydrogen bonding but not resolved in the liquid water spectrum?

To experimentally address this question, we compare the liquid water PE spectrum at high $\mathrm{O} 2 \mathrm{~s}$ cross-section with ice samples representing various local structural order in the H-bonded network, shown in Figure 2. Amorphous ice is assumed to have more structural disorder than ice close to the melting point due to defects in the H-bond network. We note that the sensitivity of the electronic structure to the change in local order and the smearing of $3 a_{1}$ in particular, supports a short-range character of the hydrogen bond involving orbital rehybridization as outlined previously for crystalline ice (13). As the disorder increases, the spectra represent a sum of increasingly varying local structures. Going from crystalline ice to liquid water, the split in the $3 a_{1}$ peak is no longer seen while the total width is retained or even becoming somewhat broader for liquid water. This is contrary to the narrowing distribution of energies that would be expected from a reduced splitting alone. (We can assume that the intrinsic width of the individual $3 a_{1}$ orbitals in the liquid remains similar to that in ice based on the width of the other valence orbitals, matching the $100 \mathrm{eV}$ spectra in Figure 1 and those reported in Ref (19) (table 2)). Instead, assuming a manifold of slightly shifted energies and intensities, a similar $3 a_{1}$ splitting for individual contributions to the total spectrum can be assumed, resulting in an increasingly smeared average as disorder increases, retaining or increasing its total energy width. Based on the gradual smearing of the $3 a_{1}$ split and the retained total energy width, we thus interpret the liquid water to possess - on average - similar electronic structure effects as those in crystalline ice. What is then the information 
content in the observed photoemission spectra in terms of local hydrogen bonding configurations?

To address the information content in the experiments, we investigate theoretically the sensitivity of the electronic structure to variations around different local hydrogen bond motifs. Figure 3 shows the local electronic structure for individual water molecules in the hydrogen-bonded network. The local electronic structure is theoretically represented by computing the individual $2 \mathrm{p}$ component (solid) and the 2 s component (dashed) of the PE spectra with the orbitals labeled according to the orbital symmetry of the free water molecule. Figure 3B shows the contribution from one molecule in the MD snapshot, displaying a typical splitting of the $3 \mathrm{a}_{1}$ states into bonding $\left(3 \mathrm{a}_{1}\right)$ and antibonding $\left(3 \mathrm{a}_{1} *\right)$ combinations. In Figure 3A, orbital plots corresponding to the different orbitals are displayed. We note the relatively local character of the $b_{1}$ orbitals where the overlap between neighboring molecules is small, in contrast to the strong delocalization of the $3 \mathrm{a}_{1}$ state into bonding and antibonding bands. Figure 3C displays a collection of spectra for 8 independently selected water molecules in a single CPMD snapshot conforming to the same local H-bond topology (D1A1 H-bond motif, see Fig 4), with the sum plotted in Fig $3 \mathrm{D}$.

As indicated by the displayed spectra, we find that most configurations show strong electronic structure changes compared to the free molecule. Most prominent is the splitting of the $3 a_{1}$ orbital due to the strong $3 a_{1}-3 a_{1}$ orbital interaction and the shift of spectral features relative to each other. However, the magnitude of the split and absolute position of the center-of-energy varies strongly with the local geometry, as expected from the different orbital overlaps for each local structure. From this we conclude that, although the summed spectrum (representing a certain hydrogen bonding motif) does not show a split of the $3 \mathrm{a}_{1}$ orbital, the underlying individual configurations are still subject to the same orbital-orbital overlap which gives rise to the well defined split in ice (13).

In Figure 4 we show summed spectra representing four different H-bond motifs in the CPMD snapshot with H-bond definition as in Ref. (47). Similar to Fig 3C-D, spectra for 8 water molecules from a single CPMD snapshot are summed for each motif. As exemplified in Fig 3C, most individual configurations show splitting of the $3 \mathrm{a}_{1}$ orbital and shifting of spectral features relative to each other. The smearing of features in the sum, in particular for the $3 \mathrm{a}_{1}$ state, results in rather featureless spectra that are similar for all motifs. This emphasizes the effect of the structural averaging and the lack of features, indicating any particular structural motif. The observed differences, e.g., in the D1A2 1b peak position, are within the statistical uncertainty of the sampling.

Figure 5 shows the full sum of all 128 configurations in a single snapshot of CPMD water and ice, representing the local $p$ character and local $s$ character of the electronic structure. The fractional contribution of the different motifs (see e.g. Fig 4) in the liquid water snapshot are 41\% D2A2, 21\% D1A2, 21\% D2A1, and 11\% D1A1, respectively, and 6\% for other H-bond motifs, which is representative for the H-bond statistics in CPMD simulations of liquid water. Also shown in the figure (Figure 5B) are the $b_{1} / b_{2}$ and $a_{1}$ 
components, extracted from the normalized experimental data in Fig 2 by subtracting a fit of Gaussian peaks to the $3 \mathrm{a}_{1}$ (or $1 \mathrm{~b}_{1}$ and $1 \mathrm{~b}_{2}$ ) intensities.

The pronounced split of the $3 \mathrm{a}_{1}$ orbital in ice is seen in the simulations in agreement with experiment (this study and Ref $(13,24,25)$ ) and other theoretical approaches $(26,27,31,32)$. As expected from the structural averaging, the $b_{1} / b_{2}$ orbitals are broadened in the CPMD liquid simulations relative to ice (elaborated in more detail by Hunt et al. (33)). The main difference in experimental PE spectra of ice and liquid water, namely the loss of observable splitting in the $3 \mathrm{a}_{1}$ peak, is also captured by the simulations. However, while the experimental liquid water spectrum shows an overall broadening of the $3 \mathrm{a}_{1}$ state similar to ice, the CPMD water has a markedly more narrow distribution. As was previously indicated, the change in shape and splitting of the occupied $3 \mathrm{a}_{1}$ orbital between the different aggregation states can be rationalized by the averaging of the electronic structure over geometrical configurations with various degrees of distortions (see e.g. Fig 3), which in the liquid results in a smearing of the $3 \mathrm{a}_{1}$ state. In terms of uncertainties in the calculations, $k$-point sampling over the Brillouin zone has been investigated previously for the occupied states and found to be unimportant; it was concluded that the $\Gamma$-point was sufficient for supercells of 32 molecules (34). All theoretical PE spectra in the present work are computed using the full 128 molecule CPMD simulation model for ice and liquid water, which should thus be sufficient for a converged occupied band structure. The narrower $3 \mathrm{a}_{1}$ peak in the simulations could indicate that CPMD generates structures with less pronounced orbital interaction compared to the experiment, although the individual spectra in Fig. 3C span the full width of the experimental $3 \mathrm{a}_{1}$ peak. A more narrow range of structures in the CPMD simulation compared to the real liquid as suggested in Ref. (47) is another possible explanation. A proper evaluation of the detailed shape and width of these peaks would, however, require a better sampling, possibly on models from path-integral simulations, as well as a more strict calculation of the ionization cross-sections in the PE spectrum.

As seen in Figures 3 and 4, PES does not lend itself particularly well to extracting information on the local geometric structure of liquid water, in sharp contrast to x-ray absorption spectroscopy $(47,50,51)$ where the difference between water and ice is much larger. This difference in sensitivity between the two techniques reflects the difference in spatial extent and thereby orbital overlap of the occupied (PES) and unoccupied (XAS) MOs. Our analysis of PES, however, indicates that the local orbital interaction involved in hydrogen bonding in liquid water is similar to that previously reported for crystalline ice (13).

\section{Conclusions}

In conclusion we have shown that experimental valence photoelectron emission spectra of liquid water show major electronic structure changes relative to the free molecule, in agreement with earlier studies. By comparing the liquid water spectrum to ice close to the melting point, amorphous ice and crystalline ice, the sensitivity to the local structural order can be observed. This is reflected in the experiment at high O2s cross-section in which $3 \mathrm{a}_{1}$ shows sensitivity to the local structural order. This sensitivity is supportive of the local orbital interaction picture of hydrogen bonding in liquid water, previously 
reported for crystalline ice (13) and suggested for liquid water (see e.g. (12)). The change in shape and splitting of the occupied $3 a_{1}$ orbital between the different aggregation states is due to the averaging of the electronic structure over geometrical structures that represent various H-bond distortions. This leads to a smearing of the $3 \mathrm{a}_{1}$ splitting in, e.g., liquid water, while maintaining the same overall broadening. Electronic structure calculations using orbital projections onto the O1s core orbital further demonstrate the effect of the structural averaging but also show a lack of spectral features that can be used to identify specific local H-bonding structures.

\section{Acknowledgements}

This work was supported by the Swedish Foundation for Strategic Research, Swedish Natural Science Research Council and National Science Foundation (US) grant CHE0089215 and CHE-0431425. DN conducted part of this work under a Postdoctoral grant from the Wenner-Gren Foundation. Portions of this research were carried out at the Stanford Synchrotron Radiation Laboratory, a national user facility operated by Stanford University on behalf of the U.S. Department of Energy, Office of Basic Energy Sciences. The work at the SSRL BL 5.1 and ALS BL 11.0.2 was supported in part by the Director, Office of Science, Office of Basic Energy Sciences, Division of Materials Sciences and Division of Chemical Sciences, Geosciences, and Biosciences of the U.S. Department of Energy at Lawrence Berkeley National Laboratory under Contract No. DE-AC0205CH11231. We would like to thank the staff at SSRL and ALS for all their assistance. 


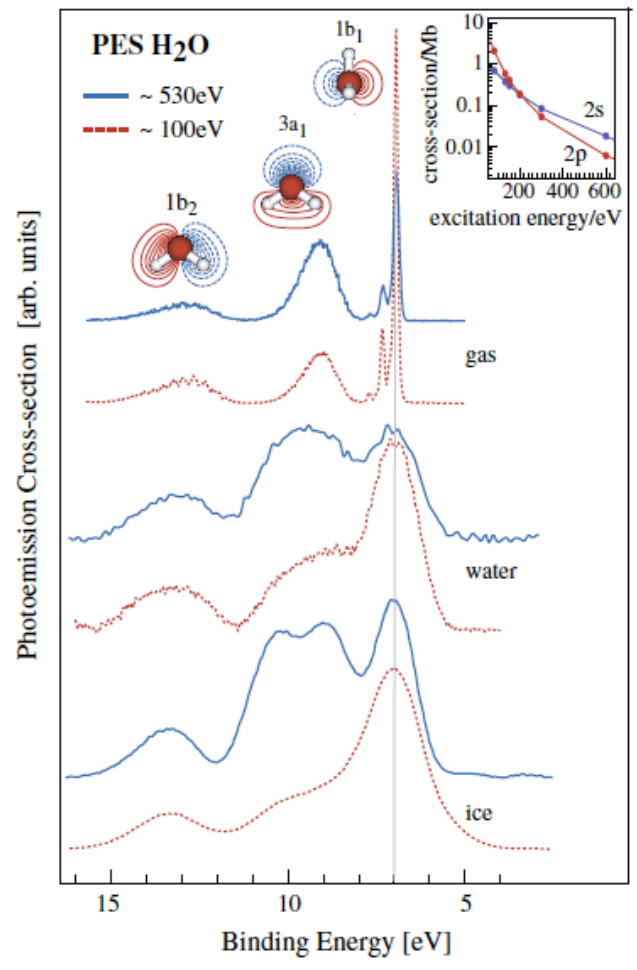

FIG 1. Valence PE spectra of gas-phase water, liquid water and crystalline ice, measured at photon energy of $100 \mathrm{eV}$ (dashed) and $530 \mathrm{eV}$ (solid). Spectra are energy normalized to the lone pair of the crystalline ice and plotted relative to the Fermi level of the $\operatorname{Pt}(111)$ substrate. The insert shows the atomic oxygen photoionization cross-section as a function of excitation energy, extracted from Ref (1) (note the logarithmic scale). 


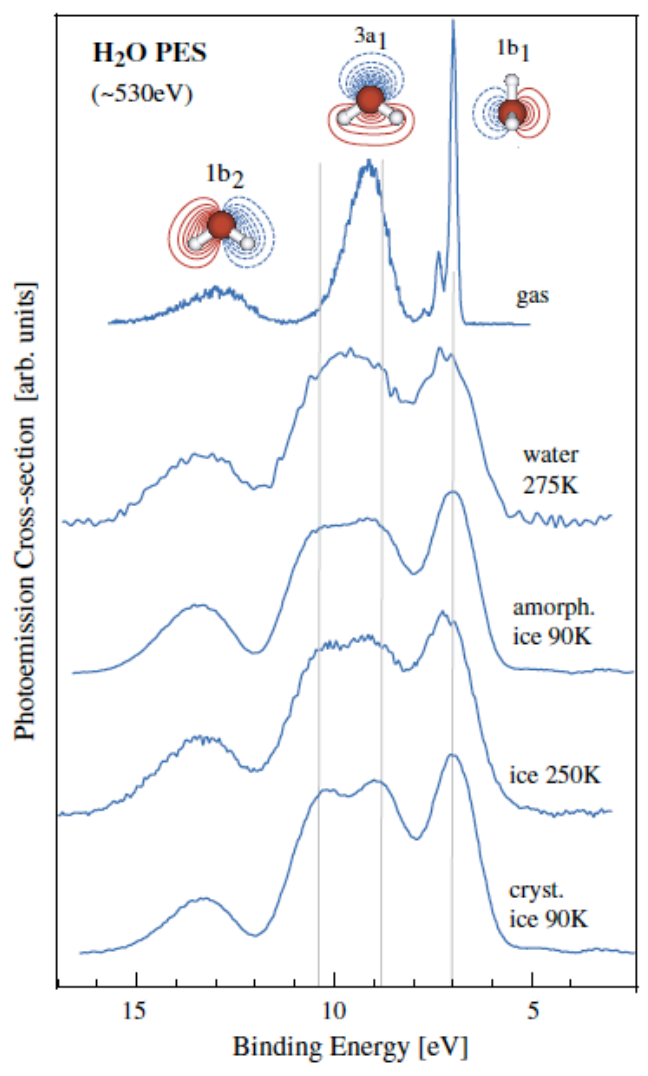

FIG 2. Valence PE spectra of water in aggregation states of increasing structural order, measured at $530 \mathrm{eV}$ photon energy (high 2 s cross-section). Vertical help-lines are shown at the energy position of the splitted $3 \mathrm{a}_{1}$ peak positions for crystalline ice. Spectra are area normalized and the energy is normalized to the lone pair peak in crystalline ice, the binding energy scale plotted relative to the Fermi energy of the $\operatorname{Pt}(111)$ substrate. 


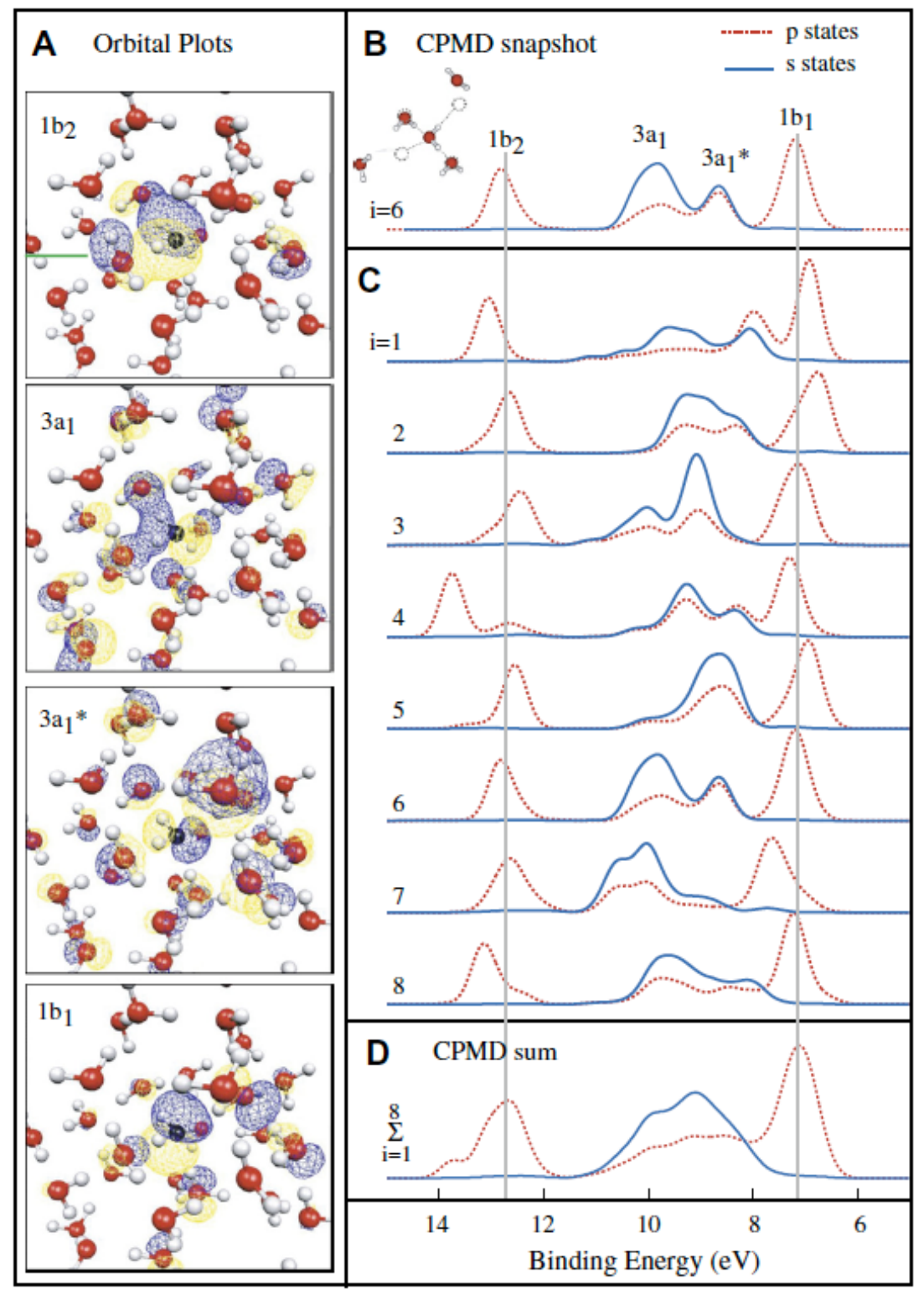

FIG 3 (A) Orbital plots for selected states taken from configuration i=6 (see B). (B,C) Individual PE spectra for molecules in a CPMD simulation model of liquid water, corresponding to the local density of states. The $2 \mathrm{p}$ (solid) and the $2 \mathrm{~s}$ (dashed) components are displayed separately and labeled according to the orbital symmetry of the free water molecule, where the $2 \mathrm{~s}$ contributions were obtained from a spectrum computed using an $r^{2}$ transition operator (see text). (C) same as (B) for 8 independently selected clusters conforming to the same local H-bond topology. (D) Sum of the spectra in (C) showing the effect of electronic structure averaging. All spectra are broadened with a Gaussian of width $0.5 \mathrm{eV}$ and a constant energy shift is applied for comparison to experimental data. 


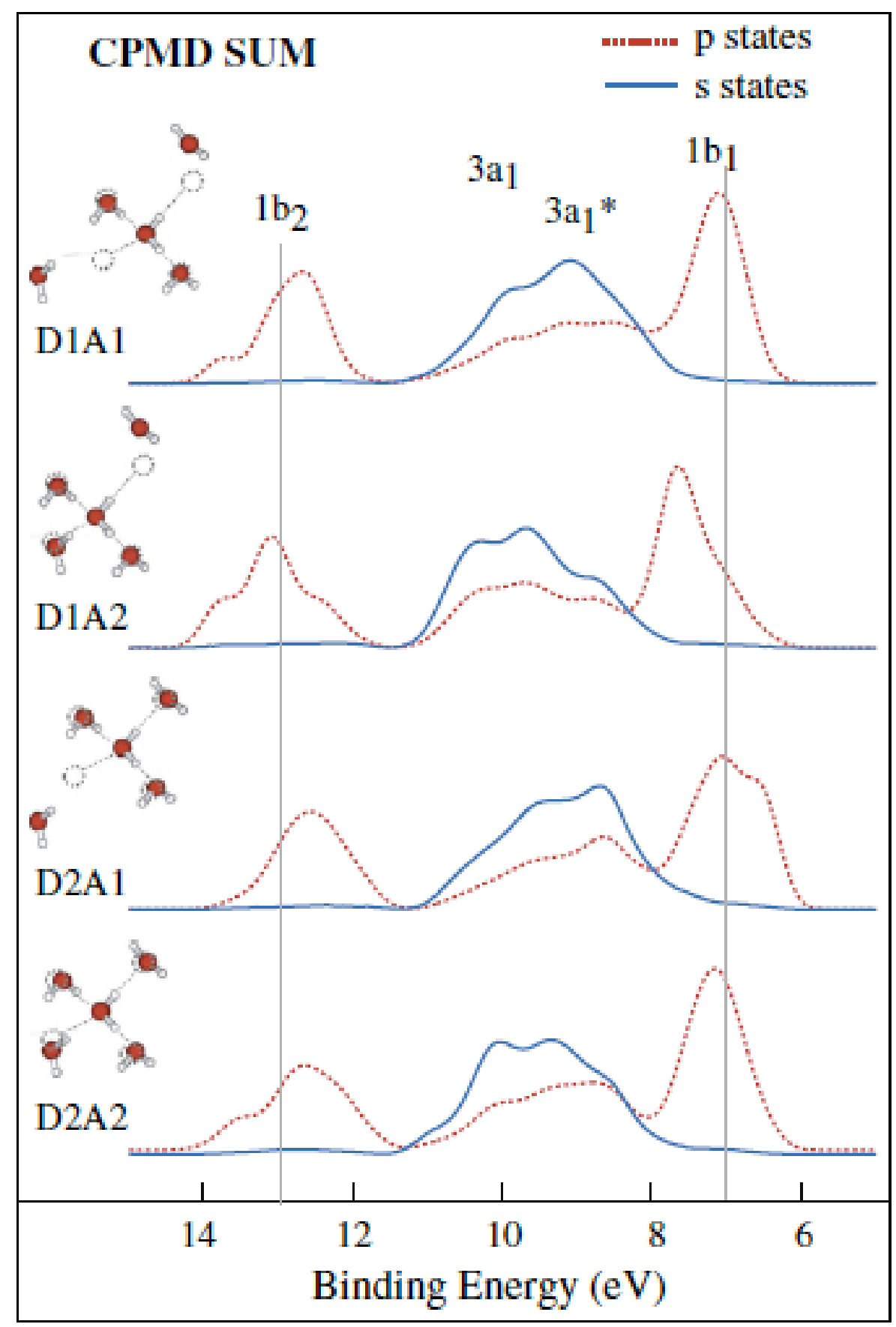

FIG 4. Sum of computed PE spectra for molecules in different hydrogen-bonding configurations selected from a CPMD simulation of liquid water according to different structural motifs in the hydrogen bonded network (see text) based on the number of strong bonds on the donor side and acceptor sides (e.g. D1A2 has one strong donor and two strong acceptors). Each spectrum is a sum of 8 individual spectra as in Fig 3C-D and the representation is otherwise the same as in FIG 3. 


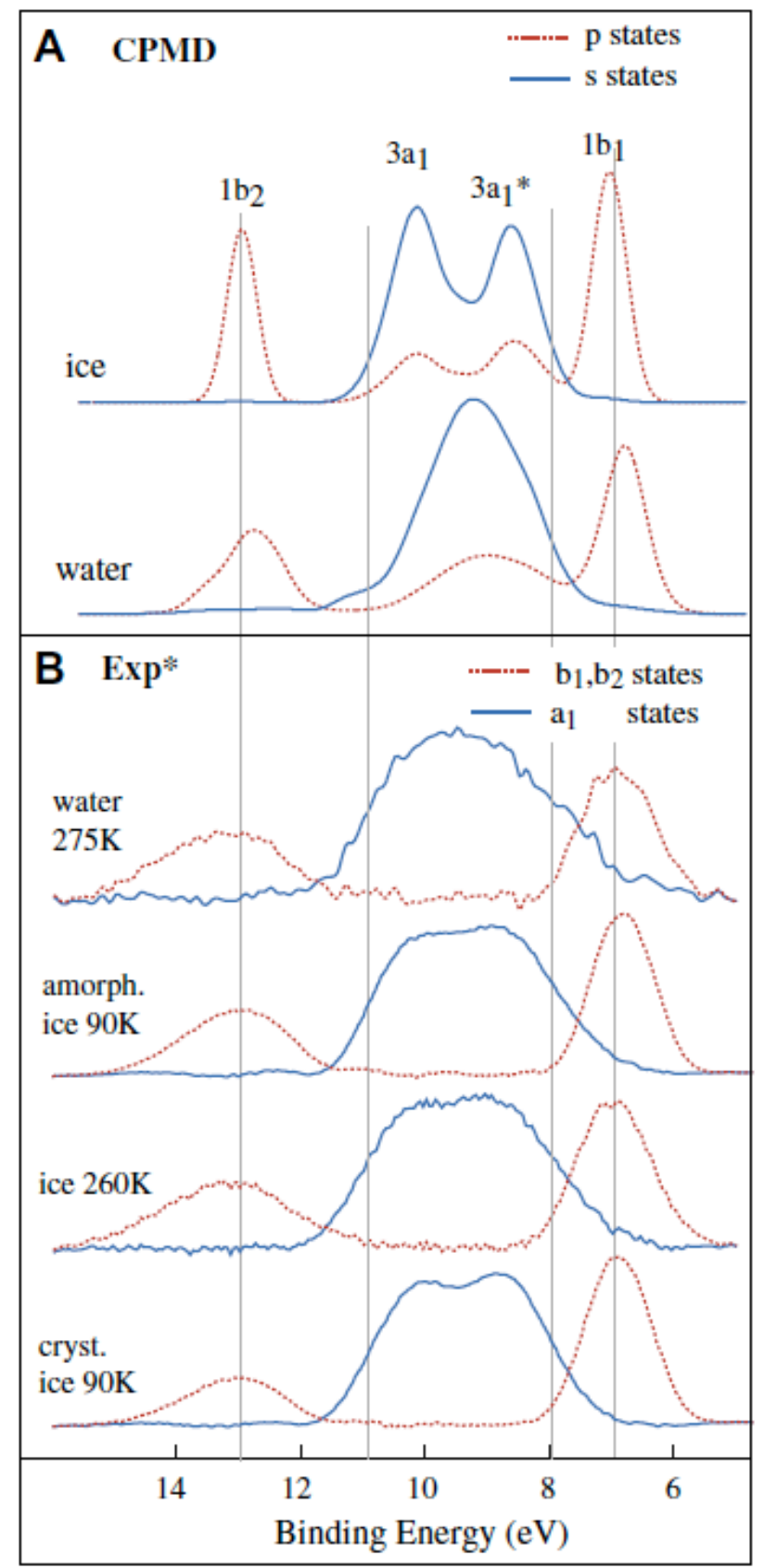

FIG 5. Comparison of simulated (A) and measured (B) electronic structure of water and ice. (A) Sum of computed PE spectra from CPMD simulations of ice and liquid water, representing the local $\mathrm{p}$ character and local s character of the states (see text and Fig. 3). (B) Experimental spectra for liquid water through crystalline ice representing the local $\mathrm{a}_{1}$ and local $b_{1} / b_{2}$ character of the states. The $a_{1}\left(b_{1} / b_{2}\right)$ component is extracted from the normalized data in Fig 2 by subtracting a fit of Gaussian peaks representing the $3 a_{1}\left(1 b_{1}\right.$ and $1 b_{2}$ ) intensities. The total width of the $3 a_{1}$ states from liquid water through crystalline ice is approximately the same $(\sim 3 \mathrm{eV})$ as indicated by the vertical lines (grey). The experimental spectra have been shifted $0.5 \mathrm{eV}$ relative to Fig 1 and Fig 2. 


\section{REFERENCES}

(1) CJ Powell, A Jablonski, NIST Electron Inelastic-Mean-Free-Path Database. National Institute of Standards and Technology, Gaithersburg, MD, 2000.

(2) L Pauling: The Nature of the Chemical Bond and the Structure of Molecules and Crystals, Cambridge University Press, Ithaka, NY, 1939.

(3) AD Buckingham, PW Fowler, JM Hutson: Theoretical-Studies of Van der Waals Molecules and Intermolecular Forces. Chemical Reviews 88 (1988) 963-88.

(4) K Morokuma: Why Do Molecules Interact - Origin of Electron Donor-Acceptor Complexes, Hydrogen-Bonding, and Proton Affinity. Accounts of Chemical Research 10 (1977) 294.

(5) PA Kollman: General Analysis of Noncovalent Intermolecular Interactions. Journal of the American Chemical Society 99 (1977) 4875.

(6) S Iuchi, S Izvekov, GA Voth: Are many-body electronic polarization effects important in liquid water? The Journal of Chemical Physics 126 (2007) 124505.

(7) LX Dang: Importance of Polarization Effects in Modeling the Hydrogen Bond in Water Using Classical Molecular Dynamics Techniques. Journal of Physical Chemistry B 102 (1998) 620-24.

(8) B Guillot: A reappraisal of what we have learnt during three decades of computer simulations on water. Journal of Molecular Liquids 101 (2002) 219.

(9) EE Dahlke, DG Truhlar: Assessment of the pairwise additive approximation and evaluation of many-body terms for water clusters. Journal of Physical Chemistry B 110 (2006) 10595.

(10) HS Frank, W-Y Wen: Structural Aspects of Ion-Solvent Interaction in Aqueous Solutions - a Suggested Picture of Water Structure. Discussions of the Faraday Society 24 (1957) 133.

(11) AE Reed, LA Curtiss, FA Weinhold: Intermolecular Interactions from a Natural Bond Orbital, Donor-Acceptor Viewpoint. Chemical Reviews 88 (1988) 899-926.

(12) FA Weinhold: Resonance character of hydrogen-bonding interactions in water and other $\mathrm{H}-$ bonded species. Advances in Protein Chemistry 72 (2006) 121.

(13) A Nilsson, H Ogasawara, M Cavalleri, D Nordlund, M Nyberg, P Wernet, LGM Pettersson: The hydrogen bond in ice probed by soft x-ray spectroscopy and density functional theory. The Journal of Chemical Physics 122 (2005) 154505.

(14) SS Xantheas: Interaction potentials for water from accurate cluster calculations. Structure and Bonding 116 (2005) 119.

(15) JED Bene, JA Pople: Intermolecular energies of small water polymers. Chemical Physics Letters 4 (1969) 426.

(16) D Hankins, JW Moskowitz, FH Stillinger: Hydrogen-bond energy nonadditivity in water. Chemical Physics Letters 4 (1970) 527.

(17) E Clementi, W KoBos, GC Lie, G Ranghino: Nonadditivity of Interaction in Water Trimers. International journal of quantum chemistry 17 (1980) 377.

(18) H Siegbahn: Electron-Spectroscopy for Chemical-Analysis of Liquids and Solutions. Journal of Physical Chemistry 89 (1985) 897.

(19) B Winter, R Weber, W Widdra, M Dittmar, M Faubel, IV Hertel: Full Valence Band Photoemission from Liquid Water Using EUV Synchrotron Radiation. Journal of Physical Chemistry A 108 (2004) 2625-32.

(20) B Winter, M Faubel: Photoemission from liquid aqueous solutions. Chemical Reviews 106 (2006) 1176.

(21) J Guo, Y Luo, A Augustsson, J-E Rubensson, C Såthe, H Ågren, H Siegbahn, J Nordgren: X-Ray Emission Spectroscopy of Hydrogen Bonding and Electronic Structure of Liquid Water. Physical Review Letters 89 (2002) 137402.

(22) K Siegbahn, C Nordling, A Fahlman, R Nordberg, K Hamrin, J Hedman, G Johansson, T Bergmark, S-E Karlsson, I Lindgren, B Lindberg: ESCA - Atomic, Molecular, and Solid State Structure Studied by Means of Electron Spectroscopy, Almqvist and Wiksells, Uppsala, 1967. O Björneholm, F Federmann, S Kakar, T Möller: Between vapor and ice: Free water clusters studied by core level spectroscopy. The Journal of Chemical Physics 111 (1999) 546. 
(24) N Mårtensson: Molecular and solid water, A comparative ESCA study. New Journal of Chemistry 1 (1977) 191-95.

(25) T Shibaguchi, H Onuki, R Onaka: Electronic Structures of Water and Ice. Journal of the Physical Society of Japan 42 (1977) 152.

(26) GP Parravicini, L Resca: Electronic States and Optical Properties in Cubic Ice. Physical Review B: Condensed Matter and Materials Physics 8 (1973) 3009.

(27) L Resca, R Resta: Energy-Bands in Cubic Ice - Abinitio Calculation Using Method of Linear Combination of Molecular-Orbitals. Physica Status Solidi B: Basic Research 81 (1977) 129.

(28) M Faubel, B Steiner, JP Toennies: Photoelectron spectroscopy of liquid water, some alcohols, and pure nonane in free micro jets. The Journal of Chemical Physics 106 (1997) 9013-31.

(29) DF Ogletree, H Bluhm, G Lebedev, CS Fadley, Z Hussain, M Salmeron: A differentially pumped electrostatic lens system for photoemission studies in the millibar range. Review of Scientific Instruments 73 (2002) 3872.

(30) D Nordlund, H Ogasawara, H Bluhm, O Takahashi, M Odelius, M Nagasono, LGM Pettersson, A Nilsson: Probing the electron delocalization in liquid water and ice at attosecond time scales. Physical Review Letters 99 (2007).

(31) VF Petrenko, IA Ryzhkin: Electron-Energy Spectrum of Ice. Physical Review Letters 71 (1993) 2626.

(32) M Ehara, M Ishida, H Nakatsuji: Theoretical study on the outer- and inner-valence ionization spectra of $\mathrm{H} 2 \mathrm{O}, \mathrm{H} 2 \mathrm{~S}$ and $\mathrm{H} 2 \mathrm{Se}$ using the SAC-Cl general-R method. The Journal of Chemical Physics 114 (2001) 8990.

(33) PA Hunt, M Sprik, R Vuilleumier: Thermal versus electronic broadening in the density of states of liquid water. Chemical Physics Letters 376 (2003) 68.

(34) D Prendergast, JC Grossman, G Galli: The electronic structure of liquid water within densityfunctional theory. The Journal of Chemical Physics 123 (2005) 014501.

(35) H Bluhm, K Andersson, T Araki, K Benzerara, GEB Jr., JJ Dynes, S Ghosal, MK Gilles, H-C Hansen, JC Hemminger, AP Hitchcock, G Ketteler, ALD Kilcoyne, EM Kneedler, JR Lawrence, GG Leppard, J Majzlam, BS Mun, SCB Myneni, A Nilsson, H Ogasawara, DF Ogletree, KH Pecher, M Salmeron, DK Shuh, B Tonner, T Tyliszczak, T Warwick, TH Yoon: Soft X-ray microscopy and spectroscopy at the molecular environmental science beamline at the Advanced Light Source. Journal of Electron Spectroscopy and Related Phenomena 150 (2006) 86.

(36) H Bluhm, DF Ogletree, CS Fadley, Z Hussain, M Salmeron: The premelting of ice studied with photoelectron spectroscopy. Journal of Physics B: Atomic, Molecular and Optical Physics 14 (2002) L227-L33.

(37) H Siegbahn, M Lundholm: A method of depressing gaseous-phase electron lines in liquid-phase ESCA spectra. Journal of Electron Spectroscopy and Related Phenomena 28 (1982) 135.

(38) I Waluyo, D Nordlund, L-Å Näslund, H Ogasawara, LGM Pettersson, A Nilsson: Spectroscopic evidence for extended crystalline ice pillar formation during isothermal heating of amorphous ice on Pt(111). Surface Science (2008).

(39) R Car, M Parrinello: Unified Approach for Molecular Dynamics and Density-Functional Theory. Physical Review Letters 55 (1985) 2471-74.

(40) J Hutter, CPMD. Copyright IBM Corp 1990-2004, Copyright MPI fur Festkörperforschung Stuttgart 1997-2001.

(41) L Kleinman, DM Bylander: Efficacious Form for Model Pseudopotentials. Physical Review Letters 48 (1982) 1425.

(42) M Odelius: Ultrafast Core-Hole-Induced Dynamics in Water Probed by X-Ray Emission Spectroscopy. Physical Review Letters 94 (2005) 227401.

(43) B Brena, D Nordlund, M Odelius, H Ogasawara, A Nilsson, LGM Pettersson: Ultra-Fast Molecular Dissociation of Water in Ice. Physical Review Letters 93 (2004) 148302.

(44) N Troullier, JL Martins: Efficient pseudopotentials for plane-wave calculations. Physical Review B: Condensed Matter and Materials Physics 43 (1991) 1993-2006.

(45) AD Becke: Density-functional exchange-energy approximation with correct asymptotic behavior. Physical Review A 38 (1988) 3098-100.

(46) C Lee, W Yang, RG Parr: Development of the Colle-Salvetti correlation-energy formula into a functional of the electron density. Physical Review B: Condensed Matter and Materials Physics 37 (1988) 785-89. 
(47) P Wernet, D Nordlund, U Bergmann, H Ogasawara, M Cavalleri, L-Å Näslund, TK Hirsch, L Ojamäe, P Glatzel, M Odelius, LGM Pettersson, A Nilsson: The structure of the first coordination shell in liquid water. Science 304 (2004) 995-99.

(48) I-FW Kuo, CJ Mundy: An ab Initio Molecular Dynamics Study of the Aqueous Liquid-Vapor Interface. Science 303 (2004) 658-60.

(49) JJ Yeh, I Lindau: Atomic subshell photoionization cross section and assymetry parameters: $1<\mathrm{Z}$ $<$ 103. Atom Data and Nuclear Data Tables 32 (1985) 1-155.

(50) SCB Myneni, Y Lou, L-Å Näslund, M Cavalleri, L Ojamäe, H Ogasawara, A Pelmenschikov, P Wernet, P Väterlein, C Heske, Z Hussain, LGM Pettersson, A Nilsson: Spectroscopic probing of local hydrogen-bonding structures in liquid water. Journal of Physics: Condenced Matter 14 (2002) L213-L19.

(51) M Odelius, M Cavalleri, A Nilsson, LGM Pettersson: X-ray absorption spectrum of liquid water from molecular dynamics simulations: Asymmetric model. Physical Review B: Condensed Matter and Materials Physics 73 (2006) 024205. 